TRANSACTIONS OF THE

AMERICAN MATHEMATICAL SOCIETY

Volume 197, 1974

\title{
SUFFICIENT SETS FOR SOME SPACES OF ENTIRE FUNCTIONS
}

BY

\author{
DENNIS M. SCHNEIDER( $\left.{ }^{1}\right)$
}

ABSTRACT. B. A. Taylor [13] has shown that the lattice points in the plane form a sufficient set for the space of entire functions of order less than two. We obtain a generalization of this result to functions of several variables and to more general spaces of entire functions. For example, we prove that if $S \subset C^{n}$ such that $d(z, S) \leq$ const $|z|^{1-\rho / 2}$ for all $z \in C^{n}$, then $S$ is a sufficient set for the space of entire functions on $\mathrm{C}^{n}$ of order less than $\rho_{0}$. The proof involves estimating the growth rate of an entire function from its growth rate on $S$. We also introduce the concept of a weakly sufficient set and obtain sufficient conditions for a set to be weakly sufficient. We prove that sufficient sets are weakly sufficient and that certain types of effective sets [8] are weakly sufficient.

1. Introduction. Let $C^{+}$be the family of all continuous positive functions on $\mathrm{C}^{n}$, let $K \subset C^{+}$, and let $E$ be the vector space of all entire functions $f$ on $\mathrm{C}^{n}$ such that $|f(z)|=O(k(z))$ as $|z| \rightarrow \infty$ for each $k \in K$. If $S \subset \mathbf{C}^{n}, f \in E$, and $k \in K$, let $\|f\|_{k, S}=\sup \{|f(z)| / k(z): z \in S\}$. When $S=\mathrm{C}^{n}$ write $\|f\|_{k}$ for $\|f\|_{k, C}$. Then \|\|$_{k}$ (resp. \|\|$_{k, S}$ ) is a seminorm on $E$, and the collection of seminorms \|\|$_{k}$ (resp. \|\|$\left._{k, s}\right), k \in K$, determines a locally convex topology on $E$ which is denoted by $(E, K)$ (resp. $(E, K(S))$ ). A subset $S$ of $\mathbf{C}^{n}$ is called a sufficient set for $E$ when the two families of seminorms $\left\{\|\|_{k}: k \in K\right\}$ and $\left\{\|\|_{k, s}: k \in K\right\}$ determine the same locally convex topology on $E$.

If there is a family $\Phi=\left\{\phi_{1} \leq \phi_{2} \leq \cdots\right\} \subset C^{+}$such that

$$
K=\left\{k \in C^{+}: \phi_{p}(z)=O(k(z)) \text { as }|z| \rightarrow \infty, p=1,2, \cdots\right\}
$$

then the family $K$ is said to be countably determined by the family $\Phi$ and we write $K=K(\Phi)$. If $S \subset \mathrm{C}^{n}$ is a set of uniqueness for the space $E$ (i.e., any function $f \in E$ which vanishes on $S$ vanishes identically), let

Presented to the Society, January 10, 1974; received by the editors September 1, 1973. $30 \mathrm{~A} 15$.

AMS (MOS) subject classifications (1970). Primary 30A98, 46E10; Secondary 30A64,

Key words and phrases. Entire function, sufficient set, weakly sufficient set, effective set, analytic variety, subharmonic function, locally convex space.

(1) This work constituted a major portion of the author's Ph. D. dissertation prepared under the direction of Professor B. A. Taylor at the University of Michigan (1973). I wish to thank Professor Taylor for his time, encouragement, and direction.

Copyright $\odot$ 1974, American Mathematical Society 


$$
E\left(\phi_{p}, S\right)=\left\{f \in E:\|f\|_{p, S}=\sup \left\{|f(z)| / \phi_{p}(z): z \in S\right\}<\infty\right\} .
$$

Then $E\left(\phi_{p}, S\right)$ is a normed linear space and $E=\bigcup_{p=1}^{\infty} E\left(\phi_{p}, S\right) .(E, \Phi)$ (resp. $(E, \Phi(S)))$ will denote the space $E$ equipped with the inductive limit topology from the Banach spaces $E\left(\phi_{p}, \mathrm{C}^{n}\right)$ (resp. the normed linear spaces $E\left(\phi_{p}, S\right)$ ). A subset $S$ of $\mathrm{C}^{n}$ is called weakly sufficient when $(E, \Phi)$ is isomorphic to $(E, \Phi(S))$ as a locally convex space.

We derive some properties of sufficient and weakly sufficient sets, and prove that if $K$ is countably determined by $\Phi$, then a sufficient set $S$ is weakly sufficient. Using a result due to B. A. Taylor [14], we show that if the class $\Phi$ satisfies some mild restrictions, then in fact $(E, K(S)) \simeq(E, K) \simeq(E, \Phi) \simeq(E, \Phi(S))$ where $\simeq$ denotes isomorphism as a locally convex space. We also prove a type of Phragmén-Lindelöf theorem which shows that if $K$ is countably determined by $\Phi$, then the growth rate of any function in $E$ is determined by its growth rate on a sufficient set. Also we obtain a relationship between V. G. Iyer's [8] notion of an effective set and weakly sufficient sets. We prove that certain types of effective sets are weakly sufficient.

Finally, we develop a method for determining discrete sufficient sets for spaces $(E, K)$ of entire functions on $\mathrm{C}^{n}$ when the functions $k \in K$ can be chosen as functions of $|z|$. Results in this direction were obtained by Taylor [13] who showed that the lattice points in the plane form a sufficient set for the space of entire functions on $\mathbf{C}$ of order less than two. Here we use methods similar to [13] and a techique due to G. Valiron [15] to obtain a generalization of this result to functions of several variables and to more general spaces of entire functions (see Theorem 5.1).

Sufficient sets were introduced by L. Ehrenpreis to study some interesting problems in analysis [2, pp. 3, 4, 13]. The duals of the spaces considered here are analytically uniform spaces $[2$, p. 8]. Thus $[2$, p. 12] if $S$ is a sufficient set for a space $(E, K)$, then every element in the dual of $(E, K)$ has a representation as a Fourier integral

$$
\int e^{i z w} d \nu(w)
$$

where $\nu$ is a bounded measure supported on the set $S$.

Also, we prove that if $S$ is a sufficient set for $(E, K)$ and if $E$ is an algebra, then any function in $E$ which is bounded on $S$ is bounded and hence reduces to a constant (Theorem 2.4). It follows from this and the fact that the lattice points in $\mathbf{C}^{n}$ form a sufficient set for the space of entire functions on $\mathbf{C}^{n}$ of order less than two that any entire function on $\mathbf{C}^{n}$ of order less than two which is bounded at the lattice points in $\mathrm{C}^{n}$ is a constant. In the case of one variable, this is a classical problem posed by Littlewood and solved by G. Polya, J. M. Whittaker, and others [16]. 
2. Sufficient sets. Let $K$ be a family of continuous positive functions on $\mathrm{C}^{n}$ which tend to infinity with $|z|$, and let $E$ be the vector space of entire functions $f$ on $\mathbf{C}^{n}$ which satisfy $|f(z)|=O(k(z))$ for each $k \in K$. For any subset $S$ of $\mathbf{C}^{n}$ define

$$
\|f\|_{k, S}=\sup \{|f(z)| / k(z): z \in S\}, \quad f \in E, k \in K .
$$

When $S=\mathbf{C}^{n}$ write $\|f\|_{k}$ for $\|f\|_{k, \mathrm{C} n}$. The seminorms \|\|$_{k}$ (resp. \|\|$_{k, s}$ ), $k \in K$, determine a locally convex topology on $E$, and $E$ equipped with this topology is denoted by $(E, K)$ (resp. $(E, K(S))$ ).

Definition 21. A subset $S$ of $\mathbf{C}^{n}$ is called a sufficient set for $E$ when the two families of seminorms $\left\{\|\|_{k}: k \in K\right\}$ and $\left\{\|\|_{k, s}: k \in K\right\}$ determine the same locally convex topology on $E$.

Thus $S$ is a sufficient set for $E$ if and only if $(E, K)$ is isomorphic to $(E, K(S)$ as a topological vector space (denoted by $(E, K) \simeq(E, K(S))$ ). For every $k \in K$ and $f \in E,\|f\|_{k, S} \leq\|f\|_{k}$ and thus the identity map $i:(E, K) \rightarrow$ $(E, K(S))$ is always continuous. Consequently, a set $S$ is a sufficient set for $E$ if and only if for every $k \in K$ there is a $k^{\prime} \in K$ and a constant $C>0$ such that for each $f \in E$

$$
\|f\|_{k} \leq C\|f\|_{k^{\prime}, s}
$$

or

$$
|f(z)| \leq k^{\prime}(z) \text { on } S \Rightarrow|f(z)| \leq C k(z) \text { on } \mathrm{C}^{n} \text {. }
$$

This last condition is a type of Phragmén-Lindelöf principle. It shows that if $S$ is a sufficient set for $E$, then the "global" growth rate of the functions in $E$ is determined by their growth rate on $S$. With some additional hypotheses on the family $K$ we will obtain a sharper theorem of this type (see Theorem 3.8).

Definition 2.2 A subset $S$ of $\mathrm{C}^{n}$ is called a set of uniqueness for a space $E$ of entire functions if $f \in E$ and $f \equiv 0$ on $S \Rightarrow f \equiv 0$.

It is easy to see that if $S$ is a sufficient set for $(E, K)$, then $S$ is a set of uniqueness for $(E, K)$. For if $f \in E, f \equiv 0$ on $S$, and $k \in K$, then there is a $k^{\prime} \in K$ and a constant $C>0$ such that $\|f\|_{k} \leq C\|f\|_{k^{\prime}, s}=0$. Consequently, $f \equiv 0$.

However, more is true. If $S$ is a sufficient set for $(E, K)$, if $E$ is an algebra, and if $f \in E$ with $|f(z)| \leq C$ on $S$, then $|f(z)| \leq C$ on $\mathbf{C}^{n}$ and hence $f$ reduces to a constant. In terms of the family $K$ of growth conditions, the assumption that $E$ is an algebra amounts to assuming that the product $k_{1} k_{2}$ belongs to $K$ whenever $k_{1}$ and $k_{2}$ belong to $K$.

To prove this result we need the following characterization of the bounded sets in $(E, K)[12$, p. 437$]$. 
Proposition 2.3. A subset $B$ of $(E, K)$ is bounded if and only if there is a function $U(z) \geq 0$ such that $|f(z)| \leq U(z)$ for all $f \in B$ and $U(z) / k(z)$ is bounded for every $k \in K$.

Proof. If $B$ is bounded, define $U(z)=\sup \{|f(z)|: f \in B\}$. Then clearly $|f(z)| \leq U(z)$ for all $f \in B$ and since $B$ is bounded and

$$
\frac{U(z)}{k(z)}=\sup \left\{\frac{|f(z)|}{k(z)}: f \in B\right\} \leq \sup \left\{\|f\|_{k}: f \in B\right\},
$$

it follows that $U(z) / k(z)$ is bounded for all $k \in K$.

Conversely, if $U(z)$ is such a function, then $\sup \left\{\|f\|_{k}: f \in B\right\} \leq\|U\|_{k}$ which is bounded for every $k \in K$. Thus $B$ is bounded.

Theorem 2.4. Let $S$ be a sufficient set for $(E, K)$ and suppose that $E$ is an algebra. Then any function $f$ in $E$ which is bounded on $S$ is bounded and bence reduces to a constant.

Proof. It is sufficient to prove the theorem for $|f(z)| \leq 1$ for $z \in S$. For if $|f(z)| \leq C$ on $S$, replace $f(z)$ by $f(z) / C$. Let

$$
B=\left\{[f(z)]^{j}: j=1,2, \ldots\right\} \text {. }
$$

Then $B$ is a bounded set in $(E, K(S))$ and so $B$ is a bounded set in $(E, K)$, since $S$ is sufficient. By Proposition 2.3, there is a function $U(z) \geq 0$ such that for all $z$,

$$
|f(z)|^{j} \leq U(z), j=1,2, \ldots \text { or }|f(z)| \leq[U(z)]^{1 / j}, j=1,2, \ldots .
$$

Holding $z$ fixed and letting $j \rightarrow \infty$ we obtain $|f(z)| \leq 1$. Since this holds for all $z$, the proof is complete.

Definition 2.5. The family $K$ is said to be countably determined if there is a family $\Phi=\left\{\phi_{1} \leq \phi_{2} \leq \cdots\right\}$ of continuous positive functions on $\mathrm{C}^{n}$ which tend to infinity with $|z|$ such that $K$ consists of the family of all continuous positive functions on $\mathrm{C}^{n}$ which satisfy $\phi_{p}(z)=O(k(z)), p=1,2, \cdots$. We will write $K=K(\Phi)$ when the family $K$ is countably determined by the family $\Phi$.

It is easy to verify $[12, \mathrm{p} .444]$ that if $K=K(\Phi)$ is countably determined, then

$$
E=\left\{f \text { entire }:|f(z)|=O\left(\phi_{p}(z)\right) \text { for some } p=1,2, \ldots\right\} \text {. }
$$

Thus, $E=\bigcup_{p=1}^{\infty} E\left(\phi_{p}\right)$ where $E\left(\phi_{p}\right)=\left\{f\right.$ entire: $\left.|f(z)|=O\left(\phi_{p}(z)\right)\right\}$. Each $E\left(\phi_{p}\right)$ is a Banach space with respect to the norm

$$
\|f\|_{p}=\sup \left\{|f(z)| / \phi_{p}(z): z \in \mathbf{C}^{n}\right\} .
$$

Consequently, $E$ may be given the inductive limit topology $[10$, p. 78$]$ from the 
sequence of Banach spaces $E\left(\phi_{p}\right)$. The space $E$ equipped with this topology is denoted by $(E, \Phi)$.

In the following proposition, $\left(E, \tau_{c}\right)$ denotes $E$ equipped with the topology of uniform convergence on compact subsets.

Proposition 2.6. (i) The seminorm topology on $E$ is finer than the topology of uniform convergence on compact subsets. That is, the identity map $i:(E, K)$ $\rightarrow\left(E, r_{c}\right)$ is continuous.

(ii) If $K=K(\Phi)$ is countably determined, then the inductive limit topology on $E$ is finer than the seminorm topology. That is, the identity map $i:(E, \Phi) \rightarrow$ $(E, K)$ is continuous.

Proof of (i). If $D$ is any compact subset of $\mathbf{C}^{n}$ and if $k \in K$, then $\sup \{|f(z)|$ : $z \in D\} \leq C_{k}\|f\|_{k}$ where $C_{k}=\sup \{k(z): z \in D\}$. This proves (i).

Proof of (ii). The identity map $i:(E, \Phi) \rightarrow(E, K)$ is continuous if and only if each of the injections $j: E\left(\phi_{p}\right) \rightarrow(E, K)$ is continuous [10, p. 79]. For every $k \in K$, and $\phi_{p} \in \Phi$,

$$
\|f\|_{k}=\sup \{f(z) \mid / k(z)\} \leq C_{p, k}\|f\|_{p}
$$

where $C_{p, k}=\sup \left\{\phi_{p}(z) / k(z)\right\}$. This proves that each injection is continuous.

Corollary 2.7. $(E, K)$ is complete.

Proof. If $\left\{f_{a}\right\}$ is a Cauchy net in $(E, K)$, then by Proposition 2.6(i) there is an entire function $f$ such that $f_{\alpha} \rightarrow f$ uniformly on compact subsets. Let $k \in K$ and $\epsilon>0$ be given. There is an $\alpha_{0}$ such that $\alpha, \beta \geq a_{0}$ implies $\left|f_{\alpha}(z)-f_{\beta}(z)\right| \leq$ $\epsilon k(z), z \in \mathbf{C}^{n}$. Since $f_{\beta} \rightarrow f$ pointwise, this implies that, if $\alpha \geq a_{0},\left|f_{a}(z)-f(z)\right|$ $\leq \epsilon k(z), z \in \mathbf{C}^{n}$. Thus $\left\|f_{a}-f\right\|_{k} \leq \epsilon$ for $\alpha \geq a_{0}$, and this proves that $f_{a} \rightarrow f$ in $(E, K)$. Finally, since $f_{a_{0}}-f \in E, f=f_{a_{0}}-\left(f_{a_{0}}-f\right) \in E$.

It is natural to ask when these two topologies on $E$ coincide. Taylor [14] proved the following theorem which gives sufficient conditions for this to be the case.

Theorem 2.8 (Taylor). Let $K=K(\Phi)$ be countably determined and let $\psi_{p}=$ $\log \phi_{p}$. Suppose that each $\psi_{p}$ satisfies the following conditions:

(i) each $\psi_{p}$ is plurisubbarmonic;

(ii) $\psi_{p+1}(z) \geq \log \left(1+|z|^{2}\right)+\widetilde{\psi}_{p}(z)$ where $\tilde{\psi}_{p}(z)=\sup \left\{\psi_{p}(z+\xi):|\xi|<1\right\}$;

(iii) for every $p>1, \psi_{p+1}-\psi_{p}$ is bounded above on every bounded set. Then the inductive limit topology and the seminorm topology on $E$ coincide. That is, $(E, K) \simeq(E, \Phi)$.

We will need the following characterization of the bounded sets in $(E, K)$ and $(E, \Phi)$. 
Proposition 2.9. Let $K=K(\Phi)$ be countably determined. The bounded sets in $(E, K)$ and in $(E, \Phi)$ coincide, and each bounded set is contained in one of the bounded sets $B_{p}=\left\{f \in E:|f(z)| \leq p \phi_{p}(z)\right\}, p=1,2, \cdots$.

Proof. [14, p. 380].

3. Weakly sufficient sets. Let $K=K(\Phi)$ be countably determined and let $S$ be a set of uniqueness (see Definition 2.2) for $(E, K)$. For each $\phi_{p} \in \Phi$,

$$
E\left(\phi_{p}, S\right)=\left\{f \in E:|f(z)|=O\left(\phi_{p}(z)\right) \text { for } z \in S\right\}
$$

is a normed linear space with respect to the norm $\|f\|_{p, S}=\sup \left\{|f(z)| / \phi_{p}(z)\right.$ : $z \in S\}$, and $E=\bigcup_{p=1}^{\infty} E\left(\phi_{p}, S\right)$. Thus $E$ may also be given the inductive limit topology determined by the spaces $E\left(\phi_{p}, S\right)$, and $(E, \Phi(S))$ will denote $E$ equipped with this topology. The next proposition states that the " $\Phi$ topology" on $E$ is finer than the "$\Phi(S)$ topology" on $E$, which is finer than the " $K(S)$ topology".

Proposition 3.1. Let $K=K(\Phi)$ be countably determined. The identity map. pings

$$
i:(E, \Phi) \rightarrow(E, \Phi(S)), \quad i:(E, \Phi(S)) \rightarrow(E, K(S))
$$

are continuous.

Proof. A base of neighborhoods of zero in $(E, \Phi(S))$ consists of the set of absolutely convex envelopes of sets of the form $\bigcup_{p=1}^{\infty} V_{p}$ where $V_{p}$ is a member of a base of absolutely convex neighborhoods of zero in $E\left(\phi_{p}, S\right)$ for each $p$ $[10$, p. 79$]$. It follows from this and the fact that the injections $E\left(\phi_{p}\right) \rightarrow E\left(\phi_{p}, S\right)$ are continuous that the injections $E\left(\phi_{p}\right) \rightarrow(E, \Phi(S))$ are continuous. Thus $i:(E, \Phi) \rightarrow(E, \Phi(S))$ is continuous.

The proof of the second assertion is the same as that of Proposition 2.6(ii).

Definition 3.2. A subset $S$ of $\mathbf{C}^{n}$ is called a weakly sufficient set for $E$ if $(E, \Phi) \simeq(E, \Phi(S))$

Theorem 33. Suppose that $K=K(\Phi)$ is countably determined and that $\Phi$ satisfies the conditions of Theorem 2.8. Then a sufficient set is a weakly sufficient set.

Proof. The theorem follows immediately from Definition 3.2, Theorem 2.8, and Proposition 3.1.

Corollary 3.4. Suppose that $K=K(\Phi)$ is countably determined and that $\Phi$ satisfies the conditions of Theorem 2.8. Then $S$ sufficient implies

$$
(E, K(S)) \simeq(E, K) \simeq(E, \Phi) \simeq(E, \Phi(S))
$$


In the following theorem we give two equivalent sufficient conditions for a a set $S$ to be weakly sufficient.

Theorem 3.5. A subset $S$ of $\mathbf{C}^{n}$ is weakly sufficient for $E$ if $S$ satisfies either of the following equivalent conditions:

(i) $E\left(\phi_{p}, S\right)$ is complete for each $p=1,2, \cdots$.

(ii) For every $p$ there is a $q$ such that $E\left(\phi_{p}, S\right) \subset E\left(\phi_{q}\right)$ and the injection is continuous.

The proof that condition (ii) implies that $S$ is weakly sufficient is the same as the proof of the first part of Proposition 3.1. Hence it only remains to show that the two conditions are equivalent. The proof will depend on Proposition 3.7 which is due to A. Grothendieck [3].

Proposition 3.6. Let $E$ be an inductive limit of a sequence $E_{p}$ of Fréchet spaces and let $u$ be a sequentially closed linear map of a Frécbet space $F$ into E. Then $u$ is continuous.

Proof. [1, p. 448].

Proposition 3.7 (Grothendieck). Let $E$ be an inductive limit of a sequence $E_{p}$ of Fréchet spaces and let $u$ be a continuous linear mapping of Fréchet space $F$ into $E$. Then there is a $q$ such that $u$ is a continuous linear mapping of $F$ into $E_{q}$.

Proof. [3, p. 198].

Proof of Theorem 3.5. First suppose that $E\left(\phi_{p}, S\right)$ is complete for all $p$. The injection $E\left(\phi_{p}, S\right) \rightarrow(E, \Phi)$ is a sequentially closed linear map. For if $f_{j} \rightarrow f$ in $E\left(\phi_{p}, S\right)$ and $f_{j} \rightarrow g$ in $(E, \Phi)$, then $f_{j} \rightarrow f$ pointwise on $\mathbf{C}^{n}$ since the inductive limit topology is finer than the topology of uniform convergence on compact subsets (Proposition 2.6(i)). Since $f_{j} \rightarrow f$ pointwise on $S$ and $S$ is a set of uniqueness, $f \equiv g$. Thus, by Proposition 3.6 and 3.7, the injection $E\left(\phi_{p}, S\right) \rightarrow$ $(E, \Phi)$ is continuous and in fact maps $E\left(\phi_{p}, S\right)$ into $E\left(\phi_{q}\right)$ for some $q$ (continuuously).

To prove the converse let $\left\{f_{j}\right\}$ be a Cauchy sequence in $E\left(\phi_{p}, S\right)$. By assumption there is a $q$ such that the injection $E\left(\phi_{p}, S\right) \rightarrow E\left(\phi_{q}\right)$ is continuous. Thus $\left\{f_{j}\right\}$ is a Cauchy sequence in $E\left(\phi_{q}\right)$ and since $E\left(\phi_{q}\right)$ is complete there is an $f \in E\left(\phi_{q}\right)$ such that $f_{j} \rightarrow f$ in $E\left(\phi_{q}\right)$. It is routine (see the proof of Corollary 2.7) to show that $f \in E\left(\phi_{p}, S\right)$ and $f_{j} \rightarrow f$ in $E\left(\phi_{p}, S\right)$. Thus $E\left(\phi_{p}, S\right)$ is complete.

Condition (ii) in Theorem 3.5 is a Phragmén-Lindelöf type condition with the set $S$ replacing the boundary of an angle. It insists that the set $S$ should be "rich" enough to determine the growth rates of the functions in $E$. For, an equivalent statement is (essentially) that for every $p$ there is a $q$ such that $|f(z)| \leq$ 
$\phi_{p}(z)$ on $S \Rightarrow|f(z)| \leq \phi_{q}(z)$ on $\mathrm{C}^{n}$. On the surface this is a finer condition on the growth rates of the functions in $E$ than that imposed by the set $S$ being a sufficient set. However in the following theorem we prove that if $K=K(\Phi)$ is countably determined, then sufficient implies condition (i), and hence condition (ii), of Theorem 3.5. We believe (but are unable to prove) that the converse of this theorem is also true.

Theorem 3.8. If $K=K(\Phi)$ is countably determined and $S$ is a sufficient set for $E$, then for every $p$ there is a $q$ such that $E\left(\phi_{p}, S\right) \subset E\left(\phi_{q}\right)$ and the injection is continuous.

Proof. By Theorem 3.5 it is enough to show that $E\left(\phi_{p}, S\right)$ is complete for all $p$. Let $\left\{f_{j}\right\}$ be a Cauchy sequence in $E\left(\phi_{p}, S\right)$. The identity map $i:(E, \Phi(S))$ $\rightarrow(E, K(S)$ ) is continuous (Proposition 3.1) and since $S$ is a sufficient set, $(E, K)$ $\simeq(E, K(S))$. Consequently, $\left\{f_{j}\right\}$ is a Cauchy sequence in $(E, K)$ and since $(E, K)$ is complete (Corollary 2.7) there is an $f \in E$ such that $f_{j} \rightarrow f$ in $(E, K)$. It is routine (see the proof of Corollary 2.7) to show that $f \in E\left(\phi_{p}, S\right)$ and $f_{j} \rightarrow f$ in $E\left(\phi_{p}, S\right)$. Thus $E\left(\phi_{p}, S\right)$ is complete.

Corollary 3.9. If $K=K(\Phi)$ is countably determined, then a sufficient set is a weakly sufficient set.

Thus we see that a sufficient set is a weakly sufficient set even if the family $\Phi$ does not satisfy the conditions of Theorem 2.8. However, in this case it does not follow that all of the topologies on the space $E$ coincide (see Corollary 3.4).

Theorem 3.10. If $S$ is a weakly sufficient set for $(E, \Phi)$ and if $E$ is an algebra, then any function in $E$ which is bounded on $S$ is bounded, and bence must reduce to a constant.

Proof. Let $f \in E$ and suppose that $|f(z)| \leq C$ on $S$. Let $g(z)=f(z) / C$ and let $B=\left\{[g(z)]^{j}: j=1,2, \cdots\right\} . B$ is a bounded subset of $E\left(\phi_{p}, S\right)$ for each $p$ and thus $B$ is a bounded set in $(E, \Phi(S))$. Since $S$ is weakly sufficient, $B$ is bounded in $(E, \Phi)$. By Proposition 2.9, there is a $p$ such that $B$ is contained in the set $B_{p}=\left\{f \in E:|f(z)| \leq p \phi_{p}(z)\right\}$. Consequently, for all $z$,

$$
|g(z)|^{j} \leq p \phi_{p}(z), j=1,2, \cdots \text { or }|g(z)| \leq\left[p \phi_{p}(z)\right]^{1 / j}, j=1,2, \ldots
$$

Holding $z$ fixed and letting $j \rightarrow \infty$ we obtain $|g(z)| \leq 1$. Since this holds for all $z$, the proof is complete.

4. The relationship between effective sets and weakly sufficient sets. The notion of an effective set is due to Iyer [8]. In this section we shall prove that certain types of effective sets are weakly sufficient.

Let $f$ be an entire function and let $M(r, D=\max \{|f(z)|:|z| \leq r\}$. The function 
$f$ is said to be of order $\rho$ if

$$
\limsup _{r \rightarrow \infty} \frac{\log \log M(r, f)}{\log r}=\rho .
$$

An entire function $f$ of order $\rho$ has type $r$ if

$$
\limsup _{r \rightarrow \infty} \frac{\log M(r, f)}{r^{\rho}}=\tau \text {. }
$$

Let $C(\rho, d)$ denote the set of all entire functions of order $\rho$ and type less than $d$. A function of order less than $\rho$ will be regarded as a function of order $\rho$ and type 0 .

Let $\left[z_{n}\right]$ denote a distinct sequence of complex numbers such that $0 \leq\left|z_{1}\right| \leq$ $\left|z_{2}\right| \leq \cdots \leq\left|z_{n}\right| \rightarrow \infty .\left[z_{n}\right]$ is said to have exponent of convergence $\rho$ if $\rho$ is the infimum of all positive numbers $a$ for which $\Sigma_{n=1}^{\infty}\left|z_{n}\right|^{-a}$ converges.

Definition 4.1. The set $\left[z_{n}\right]$ is called an effective set for $C(\rho, d)$ if the type of any function $f \in C(\rho, d)$ as determined on $\left[z_{n}\right]$ is equal to the type of $f$. That is, $\left[z_{n}\right]$ is an effective set for $C(\rho, d)$ if, for every $f \in C(\rho, d)$,

$$
\limsup _{n \rightarrow \infty} \frac{\log \left|f\left(z_{n}\right)\right|}{\left|z_{n}\right|^{\rho}}=\limsup _{r \rightarrow \infty} \frac{\log M(r, f)}{r^{\rho}} .
$$

The following theorem $[8$, p. 360$]$ gives a sufficient condition for a set $\left[z_{n}\right]$ to be an effective set for $C(\rho, d)$.

Theorem 4. 2 (Iyer). Let $\left[z_{n}\right]$ have exponent of convergence $\rho$. Then $\left[z_{n}\right]$ is an effective set for $C(\rho, d)$ if there exists an entire function $g$ with simple zeros at $z=z_{n}$ and an $b>\rho$ such that the following relations bold:

$$
\lim _{n \rightarrow \infty} \frac{\log \left|g^{\prime}\left(z_{n}\right)\right|}{\left|z_{n}\right|^{\rho}}=d,
$$

$$
\lim \frac{\log |g(z)|}{|z|^{\rho}}=d
$$

as $|z| \rightarrow \infty$ outside $\left.A(b)=\bigcup_{n}^{\infty}=1\left|z-z_{n}\right| \leq\left|z_{n}\right|^{-b}\right\}$.

Let $\left\{d_{p}\right\}$ be a strictly increasing sequence of reals with $\lim _{p \rightarrow \infty} d_{p}=d$. For each $p$ let $\phi_{p}(z)=\exp \left(d_{p}|z|^{\rho}\right)$. Then $C(\rho, d)=\bigcup_{p=1}^{\infty} E\left(\phi_{p}\right)$.

Theorem 4.3. Let $\left[z_{n}\right]$ be an effective set for $C(\rho, d)$ which satisfies the conditions of Theorem 4.2. Then $\left[z_{n}\right]$ is weakly sufficient for $C(\rho, d)$.

Before we begin the proof of this theorem, we shall state in the form of lemmas several results used by Iyer in the proof of Theorem 4.2. 
Let $\left[z_{n}\right]$ and $g$ satisfy the conditions of Theorem 4.2.

Lemma 4.4. Let $f \in C(\rho, d)$ with $\lim \sup _{n \rightarrow \infty} \log \left|f\left(z_{n}\right)\right| /\left|z_{n}\right|^{\rho}=\beta$. Choose $\eta, 0<\eta<d-\beta$, and $\lambda$ so that $d-d \lambda^{\rho} \geq \beta+\eta$. Then

$$
f(z)=\frac{g(z)}{g(\lambda z)} \bigcup_{n=1}^{\infty} \frac{f\left(z_{n}\right) g\left(\lambda z_{n}\right)}{g^{\prime}\left(z_{n}\right)} \frac{1}{z-z_{n}} .
$$

Lemma 4.5. Let $a>1$. There is a sequence $\left\{R_{j}\right\}, R_{1}<\cdots<R_{j} \rightarrow \infty$ as $j \rightarrow \infty$, and $R_{j+1} \leq a R_{j}$, such that no circle $|z|=R_{j}$ intersects any of the discs of either $A(b)$ or $A_{\lambda}(b)$ where $\lambda$ is determined in Lemma 4.4 and

$$
A_{\lambda}(b)=\bigcup_{n=1}^{\infty}\left\{\left|z-z_{n / \lambda}\right|<\left|z_{n / \lambda}\right|^{-b}\right\} \text {. }
$$

Proof of Theorem 4.3. First observe that if $S=\left[z_{n}\right]$ is an effective set for $C(\rho, d)$, then $S$ is a set of uniqueness. Consequently, by Theorem 3.5 , it is sufficient to show that, for every $p, E\left(\phi_{p}, S\right)$ is continuously injected into $E\left(\phi_{p+2}\right)$.

Let $f \in E\left(\phi_{p}, S\right)$ with $\|f\|_{p, s} \leq 1$. Then

$$
\left|f\left(z_{j}\right)\right| \leq \exp \left[d_{p}\left|z_{j}\right|^{\rho}\right], \quad j=1,2, \ldots .
$$

If $\beta=\lim \sup _{n \rightarrow \infty} \log \left|f\left(z_{n}\right)\right| /\left|z_{n}\right|^{\rho}$, then $\beta \leq d_{p}$. Choose $\eta, 0<\eta<1 / 2\left(d_{p+1}-d_{p}\right)$, and $\lambda$ so that $d-d \lambda^{\rho}=d_{p}+\eta$. Finally choose $\epsilon>0$ so small that $\epsilon<\eta$. By Lemma 4.4,

$$
f(z)=\frac{g(z)}{g(\lambda z)} \sum_{n=1}^{\infty} \frac{f\left(z_{n}\right) g\left(\lambda z_{n}\right)}{g^{\prime}\left(z_{n}\right)} \frac{1}{z-z_{n}} .
$$

Moreover, by Lemma 4.4, given $a=\left(d_{p+1} /\left(d_{p}+\eta+\epsilon\right)\right)^{-\rho}>1$, there is a sequence $\left\{R_{j}\right\}, R_{1}<R_{2}<\cdots<R_{j} \rightarrow \infty, R_{j+1} \leq a R_{j}$, such that none of the circles $|z|=R_{j}$ intersect any of the discs of either $A(b)$ or $A_{\lambda}(b)$. Using (i) and (ii) of Theorem $4.2,(4.6)$, and the choice of $\lambda$ we obtain from (4.7) that

$$
M\left(R_{j}, f\right) \leq C \exp \left[\left(d-d \lambda^{\rho}+\epsilon\right) R_{j}^{\rho}\right]
$$

for $j \geq j_{0}=j_{0}(\epsilon)$ where $C$ is a constant which is independent of $f$. Since $M(R, f)$ is an increasing function of $R$ and $R_{j+1} \leq a R_{j}$, it follows that $M(R, f) \leq C$ $\exp \left[\left(d-d \lambda^{\rho}+\epsilon\right) a^{\rho} R^{\rho}\right]$ for $R \geq R_{j_{0}}$. Since $\left(d-d \lambda^{\rho}+\epsilon\right) a^{\rho}=\left(d_{p}+\eta+\epsilon\right) a^{\rho}=d_{p+1}$, we obtain

$$
M(R, f) \leq C \exp \left[d_{p+1} R^{\rho}\right], \quad R \geq R_{j_{0}} .
$$

This establishes that if $f \in E\left(\phi_{p}, S\right)$ with $\|f\|_{p, s} \leq 1$, then $f \in E\left(\phi_{p+2}\right)$. If $f \epsilon$ $E\left(\phi_{p}, S\right)$ and $\|f\|_{p, s} \geq 1$, we apply the preceding argument to $f /\|f\|_{p, S}$ and obtain $f \in E\left(\phi_{p+2}\right)$. Thus $E\left(\phi_{p}, S\right) \subset E\left(\phi_{p+2}\right)$.

It remains to prove that the injection $E\left(\phi_{p}, S\right) \rightarrow E\left(\phi_{p+2}\right)$ is continuous. Set 


$$
A=\sup \left\{\phi_{p+1}(R) / \phi_{p+2}(R): R>0\right\}, \quad B=\sup \left\{\phi_{p+2}\left(R_{0}\right) / \phi_{p+2}(R): R>R_{0}\right\},
$$

where $R_{0}$ is the constant determined in (4.8). From (4.8) we obtain $\sup \left\{M(R, f) / \phi_{p+2}(R): R>R_{0}\right\} \leq C A$ and since

$$
\sup _{R \leq R_{0}} \frac{M(R, f)}{\phi_{p+2}(R)} \leq \sup _{R \leq R_{0}}\left[\frac{M\left(R_{0}, f\right)}{\phi_{p+2}\left(R_{0}\right)} \frac{\phi_{p+2}\left(R_{0}\right)}{\phi_{p+2}(R)}\right] \leq A B C,
$$

it follows that $\|f\|_{p+2} \leq A B C$. Thus we have proved that if $f \in E\left(\phi_{p}, S\right)$ with $\|f\|_{p, S} \leq 1$, then $\|f\|_{p+2} \leq A B C$. This establishes that the injection $E\left(\phi_{p}, S\right) \rightarrow$ $E\left(\phi_{p+2}\right)$ is continuous and completes the proof.

It is shown in $[8, \mathrm{p} .365]$ that the lattice points $z=m+i n, m, n=0, \pm 1, \pm 2$, $\cdots$, form an effective set for $C(2, \pi / 2)$ which satisfies the conditions of Theorem 4.2. Consequently we have

Corollary 4.9. The lattice points in the plane form a weakly sufficient set for $C(2, \pi / 2)$.

It will be shown in $\$ 7$ that the lattice points in the plane form a sufficient set for the space of entire functions of order less than two. Since the proof that the lattice points form an effective set for $C(2, \pi / 2)$ involves constructing a function of order 2 and type $\pi / 2$ which vanishes at the lattice points, it follows that (see Theorems 2.4 and 3.10) the lattice points do not form a weakly sufficient (nor sufficient) set for $C(2, d)$ when $d>\pi / 2$.

5. The main theorem. Let $V(r)$ be a positive increasing function of $r$ such that $V(r) \rightarrow+\infty$ as $r \rightarrow \infty$. Let $K$ be any class of continuous, positive functions $k(r)$ whose logarithms $\phi(r)=\log k(r)$ satisfy

(i) $\lim _{r \rightarrow \infty} \phi(r)=+\infty$,

(ii) $\exp \left(B_{1}^{-1} \phi\left(B_{2}^{-1} r\right)\right) \in K$ whenever $\exp (\phi) \in K$,

(iii) $\left[\phi\left(B_{4} r\right)\right]^{2} / \phi(r)=o(V(r))$ as $r \rightarrow+\infty$, where $B_{1}, B_{2}$ and $B_{4}$ are the constants appearing in (7.1).

Theorem 5.1. Let $S \subset \mathbf{C}^{n}$ such that $d(z, S) \leq$ const $|z| / \sqrt{V(2|z|)}$ for all $z \epsilon$ $\mathrm{C}^{n}$. Then $S$ is a sufficient set for $(E, K)$.

The idea behind the proof of this theorem is to obtain an estimate for $M(r, f)$ in terms of the values of $f$ on the set $S$. In particular, we shall prove (Theorem 6.16) that if $f$ is any entire function (suitably normalized) then

$$
\log M(r, f) \leq B_{1} \log M\left(B_{2} r, f, S\right)+B_{3}\left[\frac{\log M\left(B_{4}{ }^{r}, f\right)}{\sqrt{V(r)}}\right]^{2}
$$

where $M(r, f, S)=\sup \{|f(z)|:|z| \leq r, z \in S\}$ and $B_{1}, B_{2}, B_{3}, B_{4}$ depend on $V$. Roughly, 
it will follow from this inequality that $\log M(r, f) \leq \log M\left(B_{2} r, f, S\right)$ for $r \geq r_{0}$ where $r_{0}$ is independent of $f$ (see Lemma 6.14). Thus for $k \in K$,

$$
\frac{M(r, f)}{k(r)} \leq \frac{M\left(B_{2} r, f, S\right)}{k(r)} \leq \frac{M\left(B_{2} r, f, S\right)}{k_{1}\left(B_{2} r\right)}
$$

where $k_{1}(r)=k\left(B_{2}^{-1} r\right)$. Consequently, if we restrict the norms to involve only the supremum for $r \geq r_{0}$, we have $\|f\|_{k} \leq\|f\|_{k_{1}, s^{\circ}}$ Thus the set $S$ is sufficient.

The details of the proof will be given in $\$ 7$.

6. A growth estimate for entire functions. Throughout this section $V$ will denote a pure $(n-1)$-dimensional analytic variety in $\mathbf{C}^{n}$. If $a \in \mathbf{C}^{n}$ and $\delta>0$, then $V_{\delta}=\left\{z \in \mathbf{C}^{n}: d(z, v)<\delta\right\}, \quad B_{\delta}=\left\{z \in \mathbf{C}^{n}:|z|<\delta\right\}, \quad B_{\delta}(a)=\left\{z \in \mathbf{C}^{n}:|z-a|<\delta\right\}$. Finally, $\lambda_{m}$ (resp. $\sigma_{m}$ ) will denote the $m$-dimensional Hausdorff measure (resp. the $m$-dimensional surface area measure) on $\mathbf{R}^{m}$, and $\tau_{m}$ will denote the volume of the unit ball in $\mathbf{R}^{m}$.

Proposition 6.1. Let $V$ be a pure $(n-1)$-dimensional analytic variety passing through the origin in $\mathrm{C}^{n}$. Then for any $r>0, \tau_{2 n-2^{r^{2 n-2}} \leq \lambda_{2 n-2}}\left(V \cap B_{r}\right)$.

Proof. [11, p. 16].

Proposition 6.2. Let $V$ be a pure $(n-1)$-dimensional analytic variety in $\mathbf{C}^{n}$ and let $r$ and $\delta$ be positive numbers. Then

$$
\lambda_{2 n}\left(V_{\delta} \cap B_{r}\right) \leq 3^{2 n} \frac{{ }^{r} 2 n}{r_{2 n-2}} \delta^{2} \lambda_{2 n-2}\left(V \cap B_{r+2 \delta}\right) .
$$

Proof. We begin by selecting a finite number of points $z_{i} \in V \cap \bar{B}_{r+\delta}$ which have the following properties:

The balls $B_{\delta}\left(z_{i}\right)$ are pairwise disjoint.

The balls $B_{2} \delta^{\left(z_{i}\right)}$ cover $V \cap B_{r+\delta^{\circ}}$

The balls $B_{3 \delta}\left(z_{i}\right)$ cover $V_{\delta} \cap B_{r}$.

If $V \cap \bar{B}_{r+\delta}=\varnothing$, then $V_{\delta} \cap B_{r}=\varnothing$ and the result follows. Hence we may suppose that $V \cap \bar{B}_{r+\delta} \neq \varnothing$. Let $z_{1}$ be an arbitrary point of $V \cap \bar{B}_{r+\delta}$. Now select

$$
z_{2} \in\left(V \cap \bar{B}_{r+\delta}\right) \backslash B_{2 \delta}\left(z_{1}\right)
$$

and inductively select 


$$
z_{i} \in\left(V \cap \bar{B}_{r+\delta}\right) \backslash \bigcup_{j=1}^{i-1} B_{2 \delta}\left(z_{i-1}\right)
$$

By the compactness of $V \cap \bar{B}_{r+\delta}$ there are at most a finite number $k$ of points selected in this manner. It is clear from the construction that conditions (6.3) and (6.4) are satisfied. To verify condition (6.5) we note that if $\zeta \in V_{\delta} \cap B_{r}$ then $|\zeta|<r$ and there is a point $z \in V$ such that $|\zeta-z|<\delta$. Since $|z|<r+\delta$, condition (6.4) guarantees that $z \in B_{2 \delta}\left(z_{j}\right)$ for some $j$ and so $\zeta \in B_{3 \delta}\left(z_{j}\right)$.

We now complete the proof of the proposition. Since $V$ passes through the center of each of the balls $B_{\delta}\left(z_{j}\right)$, we may apply Proposition 6.1 to obtain the inequality

$$
\tau_{2 n-2} \delta^{2 n-2} \leq \lambda_{2 n-2}\left(V \cap B_{\delta}\left(z_{j}\right)\right)
$$

for $1 \leq j \leq k$. It follows from (6.5) and this inequality that

$$
\begin{aligned}
\lambda_{2 n}\left(V_{\delta} \cap B_{r}\right) & \leq \sum_{j=1}^{k} \tau_{2 n}(3 \delta)^{2 n}=3^{2 n} \frac{\tau_{2 n}}{\tau_{2 n-2}} \delta^{2} \sum_{j=1}^{k} \tau_{2 n-2} \delta^{2 n-2} \\
& \leq 3^{2 n} \frac{{ }^{\tau} 2 n}{\tau_{2 n-2}} \delta^{2} \sum_{j=1}^{k} \lambda_{2 n-2}\left(V \cap B_{\delta}\left(z_{j}\right)\right) .
\end{aligned}
$$

Using (6.3) and the fact that $B_{\delta}\left(z_{j}\right) \subset B_{r+2}, 1 \leq j \leq k$, we obtain

$$
\sum_{j=1}^{k} \lambda_{2 n-2}\left(V \cap B_{\delta}(z)\right)=\lambda_{2 n-2}\left(V \cap \bigcup_{j=1}^{k} B_{\delta}(z)\right) \leq \lambda_{2 n-2}\left(V \cap B_{r+2 \delta}\right) .
$$

Combining the last two inequalities completes the proof.

Proposition 6.6. Let $f$ be an entire function on $\mathbf{C}^{n}$ and let $V=\left\{z \in \mathbf{C}^{n}\right.$ : $f(z)=0\}$. If $r$ and $\delta$ are positive numbers, then there is a constant $A>0$ such that

$$
\lambda_{2 n}\left(V_{\delta} \cap B_{r}\right) \leq A r^{2 n-2} \delta^{2}(\log M(e(r+\delta), f)-\log |f(0)|) .
$$

Proof. By Proposition 6.2 we have

$$
\lambda_{2 n}\left(V_{\delta} \cap B_{r}\right) \leq \text { const } \delta^{2} \lambda_{2 n-2}\left(V \cap B_{r+\delta}\right)
$$

where the constant depends only on the dimension of the space. Now Jensen's formula [9] states that

$$
N(r)+\log |f(0)|=\frac{1}{\sigma_{2 n-1}} \int_{|\gamma|=1} \log |f(r y)| d \sigma_{2 n-1}(\gamma)
$$

where 


$$
N(r)=\int_{0}^{r} \frac{n(t)}{t^{2 n-1}} d t \text { and } n(t)=\left\{\begin{array}{l}
\text { the number of zeros of } f \text { in }|z| \leq t, n=1 \\
\lambda_{2 n-2}\left(V \cap B_{t}\right), n>1
\end{array}\right.
$$

Now

$$
N(e r) \geq n(r) \int_{r}^{e r} t^{-(2 n-1)} d t= \begin{cases}n(r), & n=1, \\ \frac{n(r)}{2 n-2}\left(\frac{e^{2 n-2}-1}{(e r)^{2 n-2}}\right), & n>1\end{cases}
$$

and so

$$
n(r) \leq \text { const } r^{2 n-2} N(e r)
$$

where the constant depends only on the dimension of the space.

Consequently,

$$
\begin{aligned}
\lambda_{2 n}\left(V_{\delta} \cap B_{r}\right) & \leq \text { const } \delta^{2} \lambda_{2 n-2}\left(V \cap B_{r+\delta}\right) & & \text { by (6.7) } \\
& \leq \text { const } \delta^{2} r^{2 n-2} N(e(r+\delta)) & & \text { by (6.9) } \\
& =\text { const } \delta^{2} r^{2 n-2}(\log M(e(r+\delta), f)-\log |f(0)|) & & \text { by (6.8) }
\end{aligned}
$$

and this completes the proof.

Proposition 6.10. If $f$ is analytic and satisfies $f(z) \neq 0,1$ in $|z|<\delta$, then there exist absolute constants $A$ and $l$ such that

$$
|\log | f(z)|-\log | f(0)\|\leq A+\epsilon|\log | f(0)\|
$$

for $|z| \leq \epsilon l \delta, 0<\epsilon<1$.

Proof. It is sufficient to prove the result when $f(0) \geq 0$. For the general case replace $f$ by $e^{-i \phi} f$ where $f(0)=r e^{i \phi}$. By Schottky's theorem $[5, \mathrm{p} .49]$ there exist absolute constants $A, B, C$ such that

$$
|\log | f(z) \| \leq(A+B|\log | f(0) \|)\left(\frac{\delta+z}{\delta-z}\right)^{C}
$$

for $|z|<\delta$. For $x<\delta$ the function $x \rightarrow(\delta+x) /(\delta-x)$ is increasing and so we obtain

$$
|\log | f(z)\left\|\leq A_{1}+B_{1}|\log | f(0)\right\|
$$


for $|z| \leq \delta / 2$ where $A_{1}={ }^{C} A_{1} B_{1}={ }^{C} B$. Since $f(z) \neq 0$ for $|z|<\delta, \log |f|$ is harmonic and (since $f(0)$ is real) we may apply the Poisson integral formula $[6$, p. 31] to obtain

$$
\log f(z)=\frac{1}{2 \pi} \int_{-\pi}^{\pi} \frac{r e^{i \theta}+z}{r e^{i \theta}-z} \log \left|f\left(r e^{i \theta}\right)\right| d \theta
$$

for $|z|<r=\delta / 2$. Using (6.11) to estimate the modulus of this integral we see that

$$
|\log f(z)| \leq \frac{r+|z|}{r-|z|}\left(A_{1}+B_{1}|\log | f(0) \|\right) \leq A_{2}+B_{2}|\log | f(0) \|
$$

for $|z|<r / 2=\delta / 4$ where $A_{2}=3 A_{1}, B_{2}=3 B_{1}$. By the Cauchy integral formula,

$$
\frac{d}{d z}(\log f(z))=\frac{1}{2 \pi i} \int_{|\zeta|=\delta / 4} \frac{\log f(\zeta)}{(\zeta-z)^{2}} d \zeta
$$

for $|z|<\delta / 4$. Now using (6.12) we obtain the estimate

$$
\begin{aligned}
|d \log f(z) / d z| & \leq 1 / 4 \delta\left(A_{2}+B_{2}|\log | f(0) \|\right)(\delta / 4-\delta / 8)^{-2} \\
& =(1 / \delta)\left(A_{3}+B_{3}|\log | f(0) \|\right)
\end{aligned}
$$

for $|z|<\delta / 8$ where $A_{3}=16 A_{2}, B_{3}=16 B_{2}$. Thus

$$
|\log f(z)-\log f(0)|=\left|\int_{0}^{z} \frac{d}{d \zeta} \log f(\zeta) d \zeta\right| \leq(|z| / \delta)\left(A_{3}+B_{3}|\log | f(0) \|\right)
$$
for $|z|<\delta / 8$.

Let $l=\min \left\{1 / B_{3}, 1 / 8\right\}$ and let $\epsilon$ be given with $0<\epsilon<1$. Then $\epsilon l \delta<\delta / 8$ and so we may apply (6.13) to obtain

$$
|\log f(z)-\log f(0)| \leq A_{3}+\epsilon|\log | f(0) \|
$$

for $|z|<\epsilon l \delta$. Since $\operatorname{Re}(\log f(z)-\log f(0))=\log |f(z)|-\log |f(0)|$ the assertion follows.

Lemma 6.14. Let $u, \phi, V$ be positive increasing functions of $r$ tending to infinity for which there exist constants $C_{1}$ and $C_{2}>1$ such that the following conditions bold:

(i) $\phi\left(C_{2} r\right)^{2} / \phi(r)=o(V(r))$.

(ii) $u(r)=o(\phi(r))$.

(iii) $u(r) \leq \phi(r)+C_{1}\left(u\left(C_{2} r\right) / \sqrt{V(r)}\right)^{2}$.

Then there is an $r_{0}$ (which is independent of $u$ ) sucb that $u(r) \leq 2 \phi(r)$ for $r \geq r_{0}$. 
Proof. By (i) there is an $r_{0}$ such that

$$
\phi\left(C_{2} r\right)^{2} / \phi(r) \leq V(r) / 4 C_{1}
$$

for $r \geq r_{0}$. If there is an $r>r_{0}$ with $2 \phi(r)<u(r)$ then, by (iii), $\phi(r)<$ $C_{1}\left(u\left(C_{2} r\right) / \sqrt{V(r)}\right)^{2}$. Using (6.15) and this inequality we obtain

$$
\phi\left(C_{2} r\right) \leq \frac{V(r) \phi(r)}{4 C_{1} \phi\left(C_{2} r\right)}<\frac{u\left(C_{2} r\right)^{2}}{4 \phi\left(C_{2} r\right)} .
$$

Thus $2 \phi\left(C_{2} r\right)<u\left(C_{2} r\right)$. Repeating this argument we obtain, for every $n=1,2, \cdots$, $2 \phi\left(C_{2}^{n} r\right)<u\left(C_{2}^{n} r\right)$ which contradicts (ii).

Let $\beta$ be the solution to the equation $\log |\beta|+\log |\beta-1|=1$ which is real and greater than 1. Fix $\epsilon, 0<\epsilon<1$, let $l$ be the constant appearing in Proposition 6.10 , and let $\psi(r)$ be a positive function defined for all $r>0$. Let $\widetilde{\psi}(r)=$ $\sup \{\psi(t): r / 2 \leq t \leq 3 r / 2\}$.

Theorem 6.16. Let $S \subset \mathrm{C}^{n}$ with $d(z, S) \leq \psi(|z|)$ for all $z$ and let $f$ be an entire function with $f(0)=\beta$. Then there exist constants $A_{1}>0$ depending on $n$ and a constant $A_{2}$ depending on $n$ and $\epsilon$ sucb that

$$
\begin{aligned}
\log M(r, f) \leq & (1-\epsilon)^{-1}\left(A_{1}+\log M(3 r / 2+\tilde{\psi}(r) / \epsilon l, f, s)\right) \\
& +A_{2}\left[r^{-1} \tilde{\psi}(r) \log M(e(3 r / 2+\tilde{\psi}(r)), f)\right]^{2} .
\end{aligned}
$$

Proof. Let $a \in S$ and let $D=\left\{\zeta \in \mathrm{C}^{n}:|\zeta-a| \leq|a| / 2\right\}$. Since $f$ is-entire, $\log |f|$ is subharmonic and hence satisfies the subaveraging property, i.e.,

$$
\log |f(a)| \leq(2 /|a|)^{2 n} \tau_{2 n}^{-1} \int_{D} \log |f(z)| d \lambda_{2 n}(z)
$$

If $E$ is any measurable subset of $D$ we may split this integral into the integral over $E$ plus the integral over $D \backslash E$ and obtain the estimate

$$
\log |f(a)| \leq \sup _{E} \log |f(\zeta)|+\frac{1}{r_{2 n}}\left(\frac{2}{|a|}\right)^{2 n} \lambda_{2 n}(D \backslash E) \log M\left(\frac{3|a|}{2}, f\right) .
$$

Now for $i=0,1$, set $V^{i}=\left\{z \in \mathrm{C}^{n}: f(z)=i\right\}$ and define

$$
E=\left\{\zeta \in D: V^{i} \cap B_{\delta}(\zeta)=\varnothing \text { for } i=0,1\right\}
$$

where $\delta=\tilde{\psi}(|a|) / \epsilon l$. Then $D \backslash E \subset\left(V_{\delta}^{0} \cup V_{\delta}^{1}\right) \cap B_{3|a| / 2}$ so that

$$
\lambda_{2 n}(D \backslash E) \leq \lambda_{2 n}\left(V_{\delta}^{0} \cap B_{3|a| / 2}\right)+\lambda_{2 n}\left(V_{\delta}^{1} \cap B_{3|a| / 2}\right) .
$$

Using Proposition 6.6 to estimate the right side of this inequality, we obtain 
$\lambda_{2 n}(D \backslash E) \leq$ const $|a|^{2 n-2} \delta^{2}[\log M(e(3|a| / 2+\delta), f)$

$$
+\log M(e(3|a| / 2+\delta), f-1)-\log |f(0)|-\log |f(0)-1|]
$$

However $\log (x+1) \leq 1+\log x$ for $x \geq 1$ and since $M(r, f-1) \leq M(r, \cap+1$ and $M(r, f) \geq|f(0)|=\beta>1$,

$$
\log M(e(3|a| / 2+\delta), f-1) \leq \log M(e(3|a| / 2+\delta), f)+1 .
$$

From the choice of $\beta$ and this inequality we obtain from (6.18) that

$$
\lambda_{2 n}(D \backslash E) \leq \text { const }|a|^{2 n-2} \delta^{2} \log M(e(3|a| / 2+\delta), f)
$$

where the constant depends on the dimension of the space and the choice of $\beta$. Finally this inequality together with (6.17) gives

$$
\log |f(a)| \leq \sup _{E} \log |f(\zeta)|+A\left(r^{-1} \delta \log M(e(3|a| / 2+\delta), f)\right)^{2} .
$$

We now proceed to obtain an estimate for $\sup \{\log |f(\zeta)|: \zeta \in E\}$. Let $E_{1}=$ $\{\zeta \in E:|f(\zeta)|<1\}$. If $E=E_{1}$ the theorem follows from (6.19). Thus we may suppose that $E \backslash E_{1} \neq \varnothing$. Now for any $\zeta \in E \backslash E_{1}, d(\zeta, S) \leq \psi(|\zeta|) \leq \tilde{\psi}(|a|)=\epsilon l \delta$ and so $S \cap \bar{B}_{\epsilon l \delta}(\zeta) \neq \varnothing$. Consider the function $g$ of one complex variable defined for $|z| \leq \delta$ by $g(z)=f\left(\zeta+z s_{1}\right)$ where $s_{1}=(s-\zeta) /|s-\zeta|$ and $s \in S \cap \bar{B}_{\epsilon l \delta}(\zeta)$. Applying Proposition 6.10 we obtain that there is a constant $A>1$ such that

$$
|\log | f\left(\zeta+z s_{1}\right)|-\log | f(\zeta)|\leq A+\epsilon| \log |f(\zeta)| \mid
$$

for $|z| \leq \epsilon l \delta$. But since $\zeta \in E \backslash E_{1} \log |f(\zeta)| \geq 0$ and so this inequality implies

$$
\log |f(\zeta)|-\log \left|f\left(\zeta+z s_{1}\right)\right| \leq A+\epsilon \log |f(\zeta)|
$$

or

$$
\log |f(\zeta)| \leq(1-\epsilon)^{-1}\left(A+\log \left|f\left(\zeta+z s_{1}\right)\right|\right)
$$

For $z=|s-\zeta|, \zeta+z s_{1}=\dot{s} \in S$ and $|z| \leq \epsilon l \delta$ and so

$$
\log |f(\zeta)| \leq(1-\epsilon)^{-1}(A+\log M(3|a| / 2+\epsilon l \delta, f, s)) \text {. }
$$

Consequently,

$$
\sup _{E}|f(\zeta)| \leq \sup _{E \backslash E_{1}}|f(\zeta)| \leq \frac{1}{1-\epsilon}(A+\log M(3|a| / 2+\epsilon l \delta, f, S))
$$

and combining this with (6.19) establishes the result.

7. Proof of the main theorem. Let $V(r)$ be a positive increasing function of $r$ such that $V(r) \rightarrow+\infty$ as $r \rightarrow+\infty$. If $\psi(r)=$ const $r / \sqrt{V(2 r)}$, then $\psi(r) \leq$

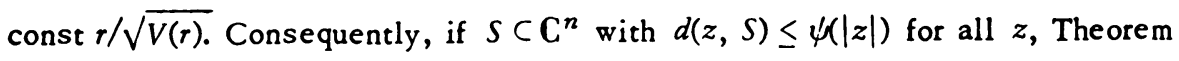


6.16 implies that there exist constants $B_{1}, B_{2}, B_{3}, B_{4}$ such that if $f$ is any entire function with $f(0)=\beta$,

$$
\log M(r, f) \leq B_{1} \log M\left(B_{2} r, f, S\right)+B_{3}\left(\frac{\log M\left(B_{4} r, f\right)}{\sqrt{V(r)}}\right)^{2}
$$

Let $K$ be any class of continuous positive increasing functions $k(r)$ whose logarithms $\phi(r)=\log k(r)$ satisfy:

(i) $\lim _{r \rightarrow \infty} \phi(r)=\infty$,

(ii) $\exp \left(B_{1}^{-1} \phi\left(B_{2}^{-1} r\right)\right) \in K$ whenever $\exp \phi(r) \in K$,

(iii) $\left[\phi\left(B_{4} r\right)\right]^{2} / \phi(r)=o(V(r))$ as $r \rightarrow \infty$, where $B_{1}, B_{2}$ and $B_{4}$ are the constants appearing in (7.1).

Theorem 5.1. Let $S$ be a subset of $\mathbf{C}^{n}$ with $d(z, S) \leq$ const $\left.|z| / \sqrt{V(2|z|}\right)$ for all $z \in \mathbf{C}^{n}$. Then $S$ is a sufficient set for $(E, K)$.

Proof. For any subset $S$ of $\mathbf{C}^{n}$ the injection $(E, K) \rightarrow(E, K(S))$ is continuous since $\|f\|_{k, s} \leq\|f\|_{k}$ for any $k \in K$. Consequently, it remains to show that the inverse is also continuous.

Following [13], given $k(r)=\exp (2 \phi(r)) \in K$, we define $k_{1}(z)=\exp \left(\phi_{1}(r)\right)$ where $\phi_{1}(r)=B_{1}^{-1} \phi\left(B_{2}^{-1} r\right)$. Then $k_{1} \in K$ and we will show that there is a constant $C>0$ such that $\|f\|_{k_{1}, s} \leq 1 \Rightarrow\|f\|_{k} \leq C$ for all $f \in(E, K)$. Since this implies that $\|f\|_{k} \leq C\|f\|_{k_{1}, s}$ the proof will be complete.

Let $f \in(E, K)$ with $\|f\|_{k_{1}, s} \leq 1$. Then $\log |f(z)| \leq \phi_{1}(z)$ for $z \in S$.

Case 1. $f(0)=\beta$. In this case we may apply Theorem 6.16 (see (7.1)) to obtain

$$
\log M(r, f) \leq B_{1} \phi_{1}\left(B_{2} r\right)+B_{3}\left(\frac{\log M\left(B_{4} r, f\right)}{\sqrt{V(r)}}\right)^{2}=\phi(r)+B_{3}\left(\frac{\log M\left(B_{4} r, f\right)}{\sqrt{V(r)}}\right)^{2} .
$$

Consequently, by Lemma 6.14, there is an $r_{0}>0$ (independent of $f$ ) such that for $r \geq r_{0}, \log M(r, f) \leq 2 \phi(r)$ or $\sup _{|z| \geq r_{0}}|f(z)| / k(z) \leq 1$. Setting $C=$ $\max \left\{k\left(r_{0}\right) / k(r): r \leq r_{0}\right\}$ we obtain

$$
\sup \left\{\frac{M(r, f)}{k(r)}: r \leq r_{0}\right\} \leq \sup \left\{\frac{M\left(r_{0}, f\right)}{k\left(r_{0}\right)} \frac{k\left(r_{0}\right)}{k(r)}: r \leq r_{0}\right\} \leq C .
$$

Consequently, $\|f\|_{k} \leq C$.

Case 2. $f(0) \neq \beta$. If $|f(0)|>\beta$, we apply Case 1 to the function $\beta f(z) / f(0)$ and deduce the same inequality.

If $|f(0)|<\beta$, we apply Case 1 to the function $f(z)-(f(0)-\beta)$ and deduce 
that $\|f(z)-(f(0)-\beta)\|_{k} \leq C$. Thus

$$
\|f\|_{k} \leq\|f(z)-(f(0)-\beta)\|_{k}+\|f(0)-\beta\|_{k} \leq C+D
$$

where $D=2 \beta \sup \{1 / k(r): r \geq 0\} \geq\|f(0)-\beta\|_{k}$.

Corollary 7.2. If $S \subset C^{n}$ with $d(z, S) \leq$ const $|z|^{1-\rho / 2}$, then $S$ is a sufficient set for the space of entire functions of order less than $\rho$.

8. A related theorem. Iyer's notion of an effective set (see $\$ 4$ ) dealt with the space $C(\rho, d)$ of entire functions of order $\rho$ and type less than $d$. A set $S=$ $\left[z_{n}\right]$ is effective for $C(\rho, d)$ if $S$ is "rich enough" so that the type of any function as determined on $S$ is equal to the type of the function. A sufficient set for the space $E_{\rho}$ of entire functions of order less than $\rho$ determines the type of a function in $E_{\rho}$ in the following sense. There is a constant $C>0$ such that if $f \in E_{\rho}$ and $f$ has type $d_{1}$ on $S$, then $f$ has type $\leq C d_{1}$. If $K$ is countably determined, then a result of this type follows from the fact that sufficient implies that for every $p$ there is a $q$ such that $E\left(\phi_{p}, S\right)$ is continuously injected into $E\left(\phi_{q}\right)$ (see Theorem 3.8). However the following proposition gives an explicit upper bound on the type of $f$ from its type on $S$.

Proposition 8.1. Let $S \subset C^{n}$ with $d(z, S) \leq|z|^{1-\rho / 2}$ for all $z$. Let $f \in E_{\rho}$ bave order $\rho_{1}<\rho$. Then

$$
\limsup _{|z| \rightarrow \infty, z \in S} \frac{\log |f(z)|}{|z|^{\rho_{1}}}=d_{1} \quad \text { implies } \limsup _{r \rightarrow \infty} \frac{\log M(r, f)}{r^{\rho_{1}}} \leq C d_{1}
$$

where $C=d_{1} B_{1} B_{2}^{\rho_{1}}$ and $B_{1}, B_{2}$ are the constants determined in (7.1).

Proof. Let $f \in E_{\rho}$ with

$$
\limsup _{|z| \rightarrow \infty, z \in S} \frac{\log |f(z)|}{|z|^{\rho_{1}}}=d_{1}, \quad \rho_{1}<\rho .
$$

Thus, given $\epsilon>0$ there is an $r_{0}$ such that $\log |f(z)| \leq\left(d_{1}+\epsilon\right)|z|^{\rho_{1}}$ for $|z| \geq$ $r_{0}, z \in S$. Letting $A=\max \left(M\left(r_{0}, f, S\right), 1\right)$ we obtain

$$
M(r, f, S) \leq A \exp \left(\left(d_{1}+\epsilon\right) r^{\rho_{1}}\right), \quad r>0 .
$$

Case 1. $f(0)=\beta$. By Theorem 6.16 and inequality (8.2) we obtain

$$
\begin{aligned}
\log M(r, f) & \leq B_{1} \log M\left(B_{2^{r}}, f, s\right)+B_{3}\left(\frac{\log M\left(B_{4}{ }^{r}, f\right)}{r^{\rho / 2}}\right)^{2} \\
& \leq B_{1} \log A+B_{1}\left(d_{1}+\epsilon\right)\left(B_{2} r\right)^{\rho}+B_{3}\left(\frac{\log M\left(B_{4} r, f\right)}{r^{\rho / 2}}\right)^{2} .
\end{aligned}
$$


Since $\log M\left(B_{4} r, f\right)=O\left({ }_{r}{ }^{1}\right)$ and $\log M\left(B_{4} r, f\right)=O\left(r^{\rho}\right)$ we obtain

$$
\underset{r \rightarrow \infty}{\lim \sup _{0}} \frac{\log M(r, f)}{\rho^{\rho_{1}}} \leq\left(d_{1}+\epsilon\right) B_{1} B_{2}^{\rho_{1}} \text {. }
$$

Case 2. $f(0) \neq \beta$. If $|f(z)|>\beta$ (resp. $|f(z)|<\beta$ ) we apply Case 1 to the function $\beta f(z) / f(0)$ (resp. $f(z)-(f(0)-\beta)$ ) and deduce the same inequality (see the proof of Theorem 5.1).

\section{BIBLIOGRAPHY}

1. R. E. Edwards, Funetional analysis. Theory and applications, Holt, Rinehart and Winston, New York, 1965. MR 36 \#4308.

2. L. Ehrenpreis, Fourier analysis in several complex variables, Pure and Appl. Math., vol. 17, Wiley-Interscience, New York, 1970. MR 44 \#3066.

3. A. Grothendieck, Espaces vectoriels topologiques, Instituto de Matemática Pura e Aplicada, Universidade de São Paulo, São Paulo, 1954. MR 17,1110.

4. W. K. Hayman, Meromorphic functions, Oxford Math. Monographs, Clarendon Press, Oxford, 1964. MR 29 \#1337.

5. M. Heins, Selected topies in the classical theory of functions of a complex variable, Athena Series: Selected Topics in Mathematics, Holt, Rinehart and Winston, New York, 1962. MR 29 \#217.

6. K. Hoffman, Banach spaces of analytic functions, Prentice-Hall Series in Modern Analysis, Prentice-Hall, Englewood Cliffs, N. J., 1962. MR 24 \#A2844.

7. L. Hörmander, Linear partial differential operators, Die Grundlehren der math. Wissenschaften, Band 116, Academic Press, New York; Springer-Verlag, Berlin, 1963. MR $28 \# 4221$.

8. V. Ganapathy Iyer, On effective sets of points in relation to integral functions, Trans. Amer. Math. Soc. 42 (1937), 358-365; correction, ibid. 43 (1938), 494.

9. P. Lelong, Propriélés métriques des variétés analytiques complexes définies par une équation, Ann. Sci. École Norm. Sup (3) 67 (1950), 393-419. MR 13, 932.

10. A. P. Robertson and W. J. Robertson, Topological vector spaces, Cambridge Univ. Press, New York, 1964. MR 28 \#5318.

11. G. Stolzenberg, Volumes, limits, and extensions of analytic varieties, Lecture Notes in Math., no. 19, Springer-Verlag, New York and Berlin, 1966. MR $34 \# 6156$.

12. B. A. Taylor, Some locally convex spaces of entire functions, Proc. Sympos. Pure Math., vol. 11, Amer. Math. Soc., Providence, R. I., 1968, pp. 431-467.

13. - Discrete sufficient sets for some spaces of entire functions, Trans. Amer. Math. Soc. 163 (1972), 207-214. MR 44 \#7269.

14. _- A seminorm topology for some (DF)-spaces of entire functions, Duke Math. J. 38 (1971), 379-385. MR 43 \#3467.

15. G. Valiron, Sur les variations du module des functions entières ou meromorphes, C. R. Acad. Sci. Paris 204 (1937), 33-35.

16. J. M. Whittaker, On the "flat" regions of integral functions of finite order, Proc. Edinburgh Math. Soc. 2 (1930), 111-128.

DEPARTMENT OF MATHEMATICS, KALAMAZOO COLLEGE, KALAMAZOO, MICHIGAN 4001

Current address: Department of Mathematics, Knox College, Galesburg, Illinois 61401 